Santos-Rego, M.A., Lorenzo-Moledo, M. \& Priegue-Caamaño, D. (2019). La Mejora de la Participación e Implicación de las Familias en la Escuela: un Programa en Acción. Revista Electrónica Interuniversitaria de Formación del Profesorado, 22(3), 93-107.

\title{
La Mejora de la Participación e Implicación de las Familias en la Escuela: un Programa en Acción
}

Miguel A. Santos Rego, Mar Lorenzo Moledo, Diana Priegue Caamaño

Universidad de Santiago de Compostela

\section{Resumen}

Tomando en consideración la relevancia de poner en marcha acciones que favorezcan la relación familia-escuela, el estudio examina la medida en que el programa socioeducativo ECOFASE ha cumplido con la eficacia prevista respecto de la mejora de los niveles de participación familiar en la escuela y de la implicación educativa de los progenitores en el hogar. En concreto, el programa se implementó en siete (7) centros de educación secundaria de la Comunidad Autónoma de Galicia, contando con la colaboración de 97 familias. Los resultados que hemos obtenido confirman que el programa, en términos generales, ha sido efectivo y ha logrado satisfacer las expectativas de las familias. De una parte, la intervención llevada a cabo ha servido para que las familias refuercen sus buenas prácticas de implicación educativa en el hogar, caso del acompañamiento en el estudio y la ayuda y apoyo prestados a sus hijas/os. De otra, ha incidido significativamente en la relación que mantienen los padres y madres con el centro escolar y, en especial, con el profesor tutor.

\section{Palabras clave}

Familia, participación en la escuela; implicación educativa; programa socioeducativo.

\section{Contacto:}

Miguel A. Santos Rego. E-mail: miguelangel.santos@usc.es. Universidad de Santiago de Compostela. Este trabajo se ha elaborado teniendo como soporte el proyecto de investigación subvencionado en convocatoria competitiva por la Xunta de Galicia, Deseño e avaliación dun programa para a mellora do rendemento educativo do alumnado inmigrante (10SEC214042PR). 


\section{The Improvement of Participation and Involvement of Families in School: a Program in Action}

\section{Abstract}

Taking into consideration the relevance of implementing actions that favor the family-school relationship, this study aims to examine the way in which the socioeducational program ECOFASE has complied with the planned effectiveness in relation to the improvement of the levels of family participation at school and parents' educational involvement at home. Specifically, the program was implemented in seven (7) secondary schools of the Autonomous Community of Galicia, with the collaboration of 97 families. The results we have obtained confirm that, in general terms, the program has been effective and has managed to meet the families' expectations. On the one hand, the intervention carried out has helped families to reinforce their good practices of educational involvement at home, as the case of accompaniment in the study as well as helping and support provided to their children. On the other, it has had a significant impact on the relationship that parents maintain with the school and, especially, with the tutor.

\section{Key words}

Family; participation at school; educational involvement; socio-educational program.

\section{Introducción}

Es posible dar cuenta de un buen número de referencias que no sólo enfatizan que las familias son un recurso clave para ayudar a promover el éxito educativo del alumnado, sino que destacan que el aprendizaje se optimiza cuando las familias y las escuelas colaboran (Santos Rego, Godás, y Lorenzo, 2012). Junto a ello, se ha constatado también que la participación de los padres y madres revierte en la calidad del centro y del trabajo docente (Llevot y Bernad, 2015), incrementa el capital social y cultural de las familias (Auduc, 2007) y permite aproximar las culturas familiar y escolar (Rodríguez-Ruiz, Martínez-González, y Rodrigo, 2016).

Tal y como se ha confirmado en trabajos previos (Santos, Lorenzo, y Priegue, 2011), el análisis del grado de implicación no puede desvincularse de variables de índole muy diversa que, en mayor o menor medida, influyen en el contacto con la escuela. En este sentido, la incursión realizada por Rodríguez, Martínez, y Rodrigo (2016), centrada en el estudio de las dificultades de las familias para participar en los centros escolares, concluye que los principales obstáculos se vinculan con las dificultades para compatibilizar el horario laboral y escolar, lo que explicaría que los padres y madres que trabajan fuera de casa informen de mayores problemas para mantener una relación estrecha con el centro escolar.

Lo cierto es que, en no pocas familias, factores como los económicos son la causa de que las necesidades vitales más urgentes estén por delante de la participación en la escuela de los hijos/as. En este sentido, las investigaciones realizadas sobre los niveles de participación 
de los progenitores de origen inmigrante constatan la importancia de la falta de tiempo o la existencia de una red de apoyo social escasa para compatibilizar sus responsabilidades familiares y laborales (Santos Rego, Godás, y Lorenzo, 2016).

Respecto del asunto que interesa, otro de los temas más preocupantes es el descenso de los niveles de implicación familiar a medida que los hijos/as avanzan en el sistema educativo. Así es que en Educación Infantil y Educación Primaria la relación entre progenitores, profesorado y centro escolar es significativamente más estrecha que en etapas posteriores (Parra, García, Gomariz, y Hernández, 2014). Para Rodríguez, Martínez, y Rodrigo (2016) las diferencias en el nivel de implicación de las familias en los centros según la etapa educativa tienen que ver con la existencia de visiones diferentes respecto de cómo entiende la familia que debe ser su relación con la escuela, en función de los estudios que cursan sus hijos/as, variable que a su vez se vincula al nivel de competencia percibido por los progenitores para ayudarles (Rivera y Milicic, 2006). En esta misma dirección apuntan Hoover-Dempsey y Sandler (2005) cuando señalan, de un lado, la influencia del sentido de eficacia y, de otro, la relevancia de la percepción que tiene la familia sobre el nivel de responsabilidad e implicación en el hogar que favorece en mayor medida los aprendizajes escolares y el éxito educativo de sus hijos/as.

Es evidente que la formación con la que cuentan los padres y madres va a determinar la ayuda que pueden prestar a sus hijos/as, sobre todo en determinadas etapas educativas, caso de la educación secundaria, donde los contenidos escolares son más complejos. En consecuencia, lo esperable es que los progenitores con niveles educativos más altos hagan uso de esa mayor capacitación para ayudar a sus hijos/as en las obligaciones escolares y supervisar las condiciones en las que se desarrolla el estudio en el hogar. Esta mejor preparación también beneficiaría la forma de intervenir en el centro escolar (Roosa et al., 2012), y situaría a los padres y madres en coyunturas más favorables para intervenir en los asuntos de la escuela.

Lo que se suele decir es que los padres y madres están implicados cuando "participan activamente en las actividades de la escuela, o ayudan de modo visible a los niños en la realización de las tareas escolares" (Santos Rego, Godás, y Lorenzo, 2016, p.11). Esta idea pone sobre la mesa dos asuntos. Por un lado, el predominio de una perspectiva básicamente instrumental de la participación (Lorenzo, Godás, y Santos Rego, 2017) y, por otro, la necesidad de diferenciar varios niveles o modalidades de colaboración entre estas dos agencias de socialización (Esptein, 2001; Reparaz y Naval, 2014).

La existencia de actitudes diferentes en las familias en función de la vía o modalidad de participación en el centro es una cuestión constatada en las investigaciones que se han realizado. Por una parte, los progenitores muestran más interés por aquellos aspectos que afectan directamente a sus hijos/as, mientras que los aspectos referidos a los asuntos colectivos de la escuela son menos importantes para ellos, o cuando menos sus niveles de implicación descienden significativamente (Garreta, 2013). Precisamente en lo tocante a su relación con los profesionales de la escuela, Hernández, García, y Gomariz (2019) subrayan que, de las dimensiones que integran la participación, la comunicación con el tutor/a de los hijos/as constituye una de las más analizadas y además es uno de los aspectos mejor valorados y que mayor satisfacción genera entre las familias. 
En el lado opuesto, las investigaciones que han tratado de desentrañar las claves del escaso interés de las familias por implicarse en las estructuras formales de participación aluden a su escasa efectividad (Silveira, 2016), a su irregularidad e ineficacia (Collet-Sabé, Besalú, Feu, y Tort, 2014), y a la consideración de que su rol es el de simples espectadores (Feito, 2007). Por poner un ejemplo, el trabajo realizado por Andrés y Giró (2016) informa de que el Consejo Escolar es un órgano inicialmente bien valorado pero que, con el tiempo, va perdiendo aceptación entre las familias porque estas perciben que están en situación de inferioridad con respecto al centro y los docentes, que son los que realmente toman las decisiones.

Lo cierto es que la necesidad de invertir mayores esfuerzos en la mejora de la valoración que las familias hacen de las estructuras formales de participación en la toma de decisiones es una cuestión que admite poca o ninguna discusión cuando se plantea la mejora de los niveles de implicación de los padres y madres en la escuela, siendo este el eje central del problema que analizamos en el presente trabajo. En este sentido, convendría avanzar, tanto en la reformulación de las propias estructuras como en proporcionar formación específica sobre la relevancia de formar parte de los procesos de toma de decisiones.

\section{Planteamiento del estudio}

\section{El Programa de Educación Familiar}

Con la mirada puesta en la mejora del rendimiento educativo del alumnado de ESO se llevó a cabo el diseño e implementación de un programa socioeducativo, que denominamos ECOFASE, compuesto, a su vez, por tres programas: Programa de Aprendizaje Cooperativo, Programa de Educación Familiar y Programa de Aprendizaje-Servicio. La estructura del Programa responde a la necesidad de intervenir en tres dimensiones clave de la intervención: Centro-aula, Centro-familia, y Centro-alumno.

Este trabajo se centra únicamente en el Programa de Educación Familiar (EF), que incluye dos subprogramas independientes pero estrechamente vinculados. Uno de ellos se centra en el trabajo con las familias de los estudiantes (Parte I), y el otro tiene como destinatarios a los propios alumnos y alumnas (Parte II). Los dos subprogramas supusieron cinco sesiones, cada una de las cuales tuvo una duración aproximada de dos horas.

Respecto del subprograma dirigido a las familias, diseñado en gran medida a partir de los resultados de una investigación previa (Lorenzo, Godás, Priegue, y Santos Rego, 2009), y con las aportaciones de Musitu y Cava (2001), lo primero que conviene resaltar es que uno de los principales objetivos fue orientar a los progenitores sobre cómo podían ayudar a sus hijos e hijas en su trayectoria escolar, teniendo en cuenta las particularidades de la adolescencia, etapa evolutiva en la que, como es sabido, las dificultades en las relaciones intrafamiliares pueden aumentar.

Los contenidos trabajados en las sesiones fueron los siguientes: ¿en qué podemos ayudar para que nuestro hijo estudie mejor? Hábitos y Técnicas de estudio; el Sistema Educativo Español: los padres y madres informados; la participación de padres y madres en el centro educativo; y las relaciones con hijos adolescentes.

Objetivos 
El objetivo general de trabajo es analizar si el programa ECOFASE incide positivamente en la implicación familiar en la educación en el hogar y en las relaciones que se establecen con el centro escolar.

Este propósito más global se concreta en dos objetivos específicos. En primer lugar, se trata de profundizar en la actuación de las familias en el hogar, atendiendo a puntos como la ayuda prestada, el control del tiempo de estudio, la supervisión de las tareas y los aspectos vinculados a la comunicación entre padres/madres e hijos en relación a las cuestiones educativas. En segundo lugar, se atiende a la relación con el centro, intentando valorar la eficacia del programa en la mejora de la participación de las familias a través de las estructuras formales.

\section{Método}

\section{Participantes y procedimiento}

En lo que tiene que ver con la muestra de estudio, participaron un total de 85 familias de alumnos/a de Educación Secundaria Obligatoria ( $1^{\circ}$ y $2^{\circ}$ curso) escolarizados en 7 centros educativos ( 6 públicos y un privado concertado) de la Comunidad Autónoma de Galicia. Los centros fueron seleccionados a partir de los datos de escolarización de niños y niñas de procedencia extranjera, y que decidieron participar de forma voluntaria después de presentar el programa tanto al equipo directivo como a los orientadores y tutores de los distintos cursos. Fueron invitadas a participar todas las familias de los centros con hijos e hijas matriculados en ambos cursos quienes, simultáneamente, estaban participando en otros módulos del programa.

Los miembros de las familias, que llegaron a 97 (en algún caso participan padre y madre), implicados en el desarrollo del programa fueron, sobre todo, las madres (81\%), representando los padres el $16.7 \%$ de la muestra. También debemos destacar la participación de abuelas (2.3\%). El 11.6\% de las familias emigraron a España, fundamentalmente, desde algún país de América Latina. Fueron seleccionadas tomando como referencia la participación de sus hijos/as en el programa. A todos ellos se les explicaron los objetivos, contenidos y metodología de trabajo. Tal y como ya se ha comentado, su decisión de colaborar en la investigación fue totalmente voluntaria.

A estas 97 personas se les administró un primer cuestionario sobre prácticas habituales a la hora de participar o implicarse en los estudios de sus hijos e hijas (pretest), que volvieron a cubrir tres meses después de finalizada la intervención (postest), en una sesión (entrevista grupal) donde las familias podían valorar, de forma abierta, su experiencia con el programa. Esta evaluación se hizo en cada uno de los 7 centros. En definitiva, optamos por un diseño pre-experimental de un solo grupo con pretest y postest.

Con la implementación del programa, el número de participantes se redujo a 79 , de los cuales el 65,8\% asistieron a la totalidad de las sesiones programadas, y el 34,2\% faltó a alguna (ver Santos Rego, Godás y Lorenzo, 2016).

\section{Instrumentos de recogida de información}

En cuanto a los instrumentos, situándonos en un enfoque metodológico mixto, además de la información obtenida a través de la entrevista grupal con las familias en la última sesión 
del programa, se utiliza un cuestionario elaborado específicamente para la investigación compuesto por escalas en formato tipo likert, y preguntas cerradas sobre perfil familiar, ambiente de estudio en el hogar y pertenencia a la AMPA. Concretamente, en este trabajo se atiende a dos de las escalas. Una primera compuesta por 17 ítems (alpha de Cronbach de .822) con los que se pretende analizar el nivel de actuación y las prácticas que el padre/madre/abuela despliegan para implicarse en la educación del niño/a; y una segunda sobre participación orgánica, con 4 ítems (alpha de Cronbach de .589). Los ítems tenían cuatro alternativas de respuesta: nada (1), poco (2), bastante (3) y mucho (4).

Con el objeto de analizar cómo se configura la estructura general en las escalas, se realizó un análisis de componentes principales con rotación Varimax. En otro trabajo previo (Santos Rego, Godás, y Lorenzo, 2016) se estudian las características psicométricas de las escalas.

Gracias al Análisis Factorial Exploratorio (AFE), siguiendo el método de extracción de Componentes Principales y rotación Varimax, se obtienen los siguientes estadísticos descriptivos: $\mathrm{KMO}=.741 ; \chi^{2}(153)=478,880, \mathrm{p}<.001$.

La extracción inicial de esta primera escala distingue cuatro factores significativos que explican un $57.13 \%$ de la varianza. El primero es "Comunicación de expectativas sobre los estudios" formado por 6 ítems (29.47\% de la varianza): Le digo claramente lo que espero de él/ella y cómo quiero que actúe; Me preocupo de que tenga éxito en los estudios; Acostumbro a animar a mi hijo/a para que consiga lo que quiere; Mi hijo/a sabe lo que espero de él/ella como estudiante; Lo/la respeto y lo/la considero importante; Cuando le mando hacer algo, le explico por qué debe hacerlo. El segundo es "Ayuda en el trabajo escolar" (11.31\% de la varianza): Normalmente asisto a los programas y charlas que ofrece la escuela a la que asiste mi hijo/a; Sé cómo manejarme en la escuela de mi hijo/a cuando quiero informarme para ayudarlo/a; Cuando mi hijo/a me pregunta o me pide ayuda en casa para resolver sus tareas escolares y puedo ayudar, lo hago; Le ayudo en el trabajo de la escuela si hay algo que no entiende; Ayudo a mi hijo/a a seleccionar las asignaturas o cursos que va a realizar cuando me pregunta. El tercero ( $8.60 \%$ de la varianza), con tres ítems, es el denominado "Control del comportamiento en la escuela" que incluye: Confía en mí para contarme lo que le pasa y lo que le preocupa; Sé lo que hace mi hijo/a en la escuela; Normalmente hago planes de futuro con mi hijo/a. Y el cuarto "Control sobre las tareas escolares" (7.75\% de la varianza): Controlo su asistencia a clase; Cuando mi hijo o hija practica algún deporte o actividad extracurricular lo/a acompaño; Controlo el tiempo que dedica en casa al estudio. También observamos una buena consistencia interna, tal y como recogen los valores a (entre .860 y .427).

Para la segunda de las escalas, se obtienen los siguientes estadísticos descriptivos: $\mathrm{KMO}=$ .616; $\chi^{2}(6)=29,849, p<.001$. La extracción inicial arrojó un único factor significativo que explica el $45.24 \%$ de la varianza. Los resultados obtenidos se muestran en la Tabla 1.

Tabla 1.

Resultados del análisis factorial de componentes principales con rotación Varimax en la Escala 2 


\begin{tabular}{lc}
\hline Asiste a las reuniones de la escuela de su hijo/a & .762 \\
\hline Conoce al profesor/a tutor/a de su hijo/a & .657 \\
\hline Asiste a las celebraciones o fiestas de la escuela de su hijo/a & .699 \\
\hline $\begin{array}{l}\text { Participa en las actividades convocadas por la AMPA de la escuela de su } \\
\text { hijo/a }\end{array}$ & .556 \\
\hline
\end{tabular}

\section{Resultados}

Todas las respuestas fueron previamente codificadas para ser tratadas con el paquete estadístico IBM SPSS Statistics 20.

A los efectos de analizar los efectos del Programa ECOFASE sobre las variables de estudio realizamos una comparación entre los datos de pretest y de postest ( $t$ de Student para muestras emparejadas).

Tabla 2.

Diferencias entre pretest y postest en los ítems de la escala 1

\begin{tabular}{|c|c|c|c|c|c|c|c|}
\hline \multirow[t]{2}{*}{ ITEMS } & \multicolumn{2}{|c|}{ Media } & \multicolumn{2}{|c|}{ DT } & \multirow[t]{2}{*}{$\mathbf{t}$} & \multirow[t]{2}{*}{ gl } & \multirow{2}{*}{$\begin{array}{l}\text { Sig. } \\
\text { (bilateral) }\end{array}$} \\
\hline & 1 & 2 & 1 & 2 & & & \\
\hline $\begin{array}{l}\text { Mi hijo/a sabe lo que espero de él/ella } \\
\text { como estudiante }\end{array}$ & 3.70 & 3.63 & .492 & .543 & 1.150 & 69 & .254 \\
\hline $\begin{array}{l}\text { Acostumbro a animar a mi hijo/a para } \\
\text { que consiga lo que quiere }\end{array}$ & 3.77 & 3.74 & .456 & .567 & .445 & 69 & .658 \\
\hline $\begin{array}{l}\text { Normalmente hago planes de futuro } \\
\text { con mi hijo/a }\end{array}$ & 3.27 & 3.36 & .760 & .660 & -.973 & 69 & .334 \\
\hline Sé lo que hace mi hijo/a en la escuela & 3.09 & 3.04 & .690 & .661 & .536 & 66 & .594 \\
\hline $\begin{array}{l}\text { Normalmente asisto a los programas } \\
\text { y charlas que ofrece la escuela a la } \\
\text { que asiste mi hijo/a }\end{array}$ & 2.99 & 3.03 & .807 & .798 & -.445 & 69 & .658 \\
\hline $\begin{array}{l}\text { Sé cómo manejarme en la escuela de } \\
\text { mi hijo/a cuando quiero informarme } \\
\text { para ayudarlo/a }\end{array}$ & 3.06 & 3.14 & .876 & .780 & -1.062 & 70 & .292 \\
\hline $\begin{array}{l}\text { Ayudo a mi hijo/a a seleccionar las } \\
\text { asignaturas o cursos que va a realizar } \\
\text { cuando me pregunta }\end{array}$ & 3.26 & 3.24 & .847 & .638 & .341 & 67 & .734 \\
\hline $\begin{array}{l}\text { Cuando mi hijo o hija practica algún } \\
\text { deporte o actividad extracurricular } \\
\text { lo/a acompaño }\end{array}$ & 2.86 & 2.92 & 1.006 & .847 & -.552 & 65 & .583 \\
\hline
\end{tabular}




\begin{tabular}{|c|c|c|c|c|c|c|c|}
\hline $\begin{array}{l}\text { Cuando mi hijo/a me pregunta o me } \\
\text { pide ayuda en casa para resolver sus } \\
\text { tareas escolares y puedo ayudar, lo } \\
\text { hago }\end{array}$ & 2.93 & 3.06 & .754 & .802 & -1.536 & 68 & .129 \\
\hline $\begin{array}{l}\text { Le ayudo en el trabajo de la escuela si } \\
\text { hay algo que no entiende }\end{array}$ & 3.11 & 3.04 & .949 & .947 & .627 & 70 & .533 \\
\hline $\begin{array}{l}\text { Cuando le mando hacer algo, le } \\
\text { explico por qué debe hacerlo }\end{array}$ & $3 \cdot 3$ & 3.17 & .667 & .721 & 1.349 & 69 & .182 \\
\hline $\begin{array}{l}\text { Confía en mí para contarme lo que le } \\
\text { pasa y lo que le preocupa }\end{array}$ & 3.05 & 2.98 & .843 & .836 & .712 & 70 & .479 \\
\hline $\begin{array}{l}\text { Lo/la respeto y lo/la considero } \\
\text { importante }\end{array}$ & 3.64 & 3.63 & .617 & .620 & .228 & 67 & .820 \\
\hline $\begin{array}{l}\text { Me preocupo de que tenga éxito en } \\
\text { los estudios }\end{array}$ & 3.80 & 3.80 & .496 & .550 & .000 & 70 & 1.000 \\
\hline $\begin{array}{l}\text { Le digo claramente lo que espero de } \\
\text { él/ella y cómo quiero que actúe }\end{array}$ & 3.70 & 3.57 & .598 & .671 & 1,381 & 69 & .172 \\
\hline Controlo su asistencia a clase & 3.78 & 3.77 & .630 & .658 & .207 & 70 & .837 \\
\hline $\begin{array}{l}\text { Controlo el tiempo que dedica en } \\
\text { casa al estudio }\end{array}$ & $3 \cdot 35$ & 3.45 & .946 & .692 & -.961 & 70 & .340 \\
\hline
\end{tabular}

Como se puede observar en los datos de la tabla, no se producen diferencias estadísticamente significativas entre la situación de pretest y postest. Los padres consideran ya antes del programa que comunican a los hijos sus expectativas sobre los estudios, y se preocupan, sobre todo, de que tengan éxito en los mismos. Es en la ayuda en el trabajo escolar donde las medias son más bajas, aunque siempre por encima de la media teórica de la escala. Concretamente, si bien se mejora después de la intervención, es en la prestación de ayuda, si el progenitor puede hacerlo, en casa para resolver tareas escolares, y en la participación en programas y charlas del centro, donde las familias parecen implicarse menos. Controlan la asistencia a clase, pero ya no tanto el tiempo dedicado al estudio en el hogar, y aún menos se acompaña al niño o niña a actividades extraescolares. Finalmente, consideran que sí controlan el comportamiento de sus hijos/as en la escuela.

Estos datos nos ponen sobre la pista de si este tipo de programas llega realmente a las familias que más lo necesitan, o por el contrario, son ya las familias con un nivel de compromiso alto las que acaban por participar activamente. Es por ello que conviene estudiar de forma más pormenorizada cuáles son las variables familiares que inciden de forma significativa en los resultados del programa. Así, por ejemplo, los resultados son muy distintos para aquellas familias que participaron en todas las sesiones del programa (Santos Rego, Godás, y Lorenzo, 2016). 
Es en la escala de participación orgánica donde sí se detectan diferencias estadísticamente significativas. Llama la atención el bajo conocimiento que tienen del profesor/a tutor de sus hijos/as, que mejora significativamente con la intervención. También la presencia de los padres en celebraciones o fiestas escolares se ha visto muy favorecida con el programa. Debe destacarse la baja implicación de las familias en las Asociaciones.

Tabla 3.

Diferencias entre pretest y postest en los ítems de la escala 2

\begin{tabular}{|c|c|c|c|c|c|c|c|}
\hline \multirow[t]{2}{*}{ ITEMS } & \multicolumn{2}{|c|}{ Media } & \multicolumn{2}{|c|}{ DT } & \multirow[t]{2}{*}{$\mathbf{t}$} & \multirow[t]{2}{*}{ gl } & \multirow{2}{*}{$\begin{array}{l}\text { Sig. } \\
\text { (bilateral) }\end{array}$} \\
\hline & 1 & 2 & 1 & 2 & & & \\
\hline $\begin{array}{l}\text { Asiste a las reuniones de la escuela de su } \\
\text { hijo/a }\end{array}$ & 3.15 & 3.20 & .804 & .839 & $-.445^{-}$ & 70 & .658 \\
\hline Conoce al profesor/a tutor/a de su hijo/a & 2.79 & 3.20 & 1.034 & .894 & -4.611 & 69 & .000 \\
\hline $\begin{array}{l}\text { Asiste a las celebraciones o fiestas de la } \\
\text { escuela de su hijo/a }\end{array}$ & 1.84 & 2.16 & .914 & .914 & -2.147 & 43 & .037 \\
\hline $\begin{array}{l}\text { Participa en las actividades convocadas } \\
\text { por la AMPA de la escuela de su hijo/a }\end{array}$ & 1.67 & 1.68 & .914 & .983 & -.127 & 59 & .899 \\
\hline
\end{tabular}

En la entrevista grupal las familias hacen alusión a aquellos aspectos en los que consideran que el programa ha incidido en mayor medida:

- Les permitió reforzar la forma de actuar en el hogar, lo que repercutió positivamente en las relaciones familiares: diálogo, control y acompañamiento en el estudio, conocimiento de lo que están haciendo los hijos/as, percepción por parte de los chicos de que lo que hacen es de interés para sus padres...

- Se incrementó el nivel de empatía y reflexión en la relación con sus hijos/as.

- Les sirvió para comprender y ayudar mejor a sus hijos/as en el estudio y cambiaron algunas de sus prácticas de relación.

- Consideran que los resultados se percibirán en mayor medida a largo plazo, pero ahora comprenden mejor las dificultades de sus hijos/as y tienen mayor seguridad a la hora de actuar.

Para analizar los resultados de este programa, también es conveniente reparar en la satisfacción de las familias. Una prueba de la valoración que hacen los progenitores es que más del $65 \%$ de ellos asistieron a todas las sesiones, lo que se explica por el alto nivel de satisfacción de las familias con la intervención realizada. Concretamente, se evalúa con los progenitores el programa en su conjunto, y también los contenidos, actividades y profesionales encargados: 
a) El programa en general: consideran que es útil, suficiente, apropiado, divertido, descansado, activo, corto, con un horario adecuado, ordenado y claro. En este apartado, los padres hacen observaciones referidas, no solo al horario, sino al momento en que se lleva a cabo el programa (a partir de febrero), incidiendo en la necesidad de que se desarrolle al comienzo del curso:

"Se debe hacer antes para que los niños puedan sacar mayor provecho" (Familia 9. Centro $\left.n^{\circ} 1\right)$.

"Debería haber sido al principio del curso y no al final" (Familia 14. Centro ${ }^{\circ} 1$ ).

"Deberían proponer más cursos durante el año o más tiempo; en un momento más adecuado para los niños "(Familia 31. Centro $n^{\circ} 3$ ).

b) Los contenidos: son suficientes, comprensibles, apropiados y con aplicación práctica. Al respecto, hacen observaciones importantes que deben tenerse en cuenta en futuras implementaciones del Programa:

- Reclaman que deben ampliarse, incidiendo, principalmente, en la necesidad de profundizar en la cuarta sesión, la dedicada a tratar la adolescencia y, por lo tanto, también el tiempo:

"Me gustaría que la sesión dedicada a la adolescencia fuera algo más extensa y que tratara con algo más de tiempo los aspectos psicológicos de esta etapa de la vida (Familia 6. Centro No. 1).

"Debería tener más contenido y más tiempo, toda la ayuda es poca" (Familia 24. Centro No. 3).

"Me gustaron mucho las charlas y lo que hizo (se refiere a la profesora del Programa) pero habría que explicarlo con más tiempo, hubo cosas que le noté a mi hija que le ayudaron a ella..., pero fue educativo e importante" (Familia 33. Centro ${ }^{\circ} 3$ ).

- Las familias también hacen recomendaciones, como compartir sesiones con los niños/as y la necesidad de que el programa sea aún más práctico:

"Eché en falta una sesión conjunta con los niños, tal vez sería necesaria alguna otra sesión" (Familia 60. Centro $n^{\circ} 3$ ).

"Estaba bien, pero tenía que hacerse más a menudo y más práctico" (Familia 64. Centro $\left.n^{\circ} 3\right)$.

"Cosas prácticas de métodos de estudio con padres e hijos juntos" (Familia 65. Centro $\left.n^{\circ} 3\right)$.

c) Las actividades: padres y madres las definen como comprensibles, claras, apropiadas, activas y divertidas.

d) Los profesores: las familias están de acuerdo en que los profesionales implicados fueron cercanos, activos, claros, comprensibles y agradables. 
A pesar de estos resultados globales, se considera, desde la interpretación de los datos cuantitativos y cualitativos, que la intervención ha sido positiva, teniendo en cuenta, además, el corto espacio de tiempo transcurrido entre ambas condiciones, lo que hace difícil profundizar en tales resultados.

\section{Discusión y Conclusiones}

El primer aspecto que conviene resaltar es que las familias participantes en el programa decidieron voluntariamente implicarse en su desarrollo, lo que ya de partida les atribuye un cierto interés por contribuir activamente a la mejora de las posibilidades de éxito académico de sus hijos/as. Además, en su mayor parte han mostrado una asistencia regular a las sesiones lo que refuerza la tesis de que, a priori, el programa ha conseguido implicar a familias que tienen un nivel de compromiso con la participación en la escuela superior a la media.

En segundo lugar, los resultados alcanzados ponen de manifiesto la existencia de un impacto diferente del programa a dos niveles (Pomerantz, Moorman y Litwack, 2007): de una parte, podemos hacer referencia a la eficacia del programa en las variables que asociamos a la implicación educativa parental en el hogar, y, de otra, a su eficacia para mejorar la relación que, desde un punto de vista más formal, mantienen los progenitores con la escuela.

En cuanto a los aspectos que integran el primero de los bloques, el análisis cuantitativo no refleja la existencia de mejoras estadísticamente significativas. No obstante, los resultados de pretest ya mostraban puntuaciones elevadas en buena parte de las variables contempladas, por lo que, teniendo en cuenta el escaso periodo de tiempo transcurrido entre ambas medidas, es difícil observar mejoras destacables. Así, tal y como ya se comentado, los progenitores que participan en la implementación del programa dan cuenta de buenas prácticas a la hora de implicarse en las cuestiones educativas en el hogar. Alcanzan resultados muy favorables en variables que la investigación ha constatado que son claves, caso de la comunicación de las expectativas sobre lo que se espera de los hijo/as, la preocupación por los estudios y el apoyo brindado (Hill y Tyson, 2009).

Paralelamente, si se tienen en cuenta las conclusiones recogidas en las entrevistas grupales llevadas a cabo en la última sesión, los participantes hacen hincapié en que el programa les ayudó a ser más conscientes de la importancia de implicarse en los estudios de sus hijos/as y a entender que abordar desde el diálogo y la comunicación con los hijos/as los asuntos educativos repercute positivamente en su trayectoria escolar. En cuanto a este asunto, conviene hacer una breve referencia a los hallazgos derivados de las investigaciones que han atendido a la influencia de los estilos educativos familiares en el rendimiento académico (Inam, Nomaan, y Abiodullah, 2016). Si bien todavía no existe una asociación clara sobre qué modelos educativos muestran una incidencia más favorable en la trayectoria académica de los estudiantes, lo que sí parece constatarse es que, para el alumnado, sentir el apoyo de los progenitores tiene un efecto mucho más favorable en el aprendizaje que el establecimiento en el hogar de mecanismos de control estrictos (Jeynes, 2011).

Retomando el análisis de la incidencia que ha tenido el programa en la relación de los progenitores con el centro, las diferencias entre pretest-postest muestran una mayor 
eficacia del programa en esta dimensión. Conviene recordar que precisamente la implicación de las familias en las estructuras formales es la modalidad participativa que la investigación ha demostrado ser más endeble (Santos Rego, Godás, y Lorenzo, 2016).

Los resultados alcanzados respecto de la participación en las actividades que se organizan desde las AMPA concuerdan con los derivados de otros trabajos previos realizados hace más de una década, caso del liderado por Garreta (2008), y de algunos estudios más recientes, como el llevado a cabo por Llevot y Bernad (2015). Justamente en este trabajo se afirma que el $75 \%$ de las familias de los centros están inscritas en tales asociaciones, siendo mucho más bajo el porcentaje de padres y madres (cerca del 50\%) que suele asistir a las actividades que se organizan y a las reuniones que se convocan. Es previsible, entonces, la escasa disposición de las familias para asumir responsabilidades en las juntas directivas puesto que, por lo general, su implicación se reduce a la colaboración en acciones puntuales que afectan directamente a sus hijos/as. Idéntica situación se detecta cuando se analizan las cifras de progenitores que participan en las elecciones al Consejo Escolar que, en general, no suele superar el 12 \% (Andrés y Giró, 2016).

Quizás sea precisamente la escasa utilidad percibida por las familias el principal obstáculo para participar más activamente en estas estructuras, lo que, a su vez, explicaría que los mejores índices se registren en las vías de participación que permiten resolver asuntos que afectan de manera particular a los hijos/as.

En este caso, el programa ha servido para que las familias mejoren significativamente las relaciones que mantienen con el profesor-tutor, un dato que parecía preocupante tras conocer que la medida de pretest apuntaba a una relación bastante mejorable con este profesional que, no olvidemos, es el referente en la relación centro-familia.

Lo cierto es que al analizar los datos de la primera escala que, como decíamos, daban cuenta de la existencia de un buen nivel de implicación en la educación, sorprende la escasa participación de las familias en las celebraciones de la escuela. Esta es una variable que, por lo que informan las familias, mejora de manera significativa tras haber asistido a las distintas sesiones del programa. En todo caso, los resultados no son aún los deseables. Probablemente tengan que ver con esa tendencia más global de los progenitores a implicarse menos en el centro a medida que los hijos/as avanzan en el sistema educativo y a la visión generalizada de que la educación secundaria tiene una finalidad más estrictamente académica, en la que las familias tienen poco o nada que aportar.

No sorprende, por ello, que la invitación directa del profesorado a las familias para que se impliquen en el centro escolar sea una de las variables más influyentes en la decisión de participar (Anderson y Minke, 2007). Y más aún en educación secundaria, donde el sentido de pertenencia al centro escolar podría registrar los índices más bajos. Precisamente algunos trabajos atribuyen al sentido de pertenencia una importancia más relevante en los niveles de participación que la otorgada a las variables socioeconómicas, la nacionalidad o el nivel de estudios, equiparándose a la importancia otorgada al clima educativo familiar y a las expectativas de las familias sobre el rendimiento (Castro et al., 2014).

En definitiva, sin dejar de reconocer las limitaciones que puede haber tenido el desarrollo del programa en los centros, entre ellas el escaso tiempo dedicado al trabajo con las familias, lo cierto es que tanto los datos derivados de este trabajo como muchos otros a los que se ha hecho referencia, apuntan de manera clara a la existencia de un problema serio 
en las estructuras de participación de las familias en los centros escolares. Está claro, por tanto, que además de estar lejos de alcanzar los propósitos para los que han sido configuradas, la percepción entre la comunidad educativa respecto de la utilidad de las asociaciones requiere de una reformulación en profundidad de su sentido y cometido. Algo que, necesariamente, debe estar liderado por las propias familias.

\section{Referencias}

Anderson, K. J. y Minke, K. M. (2007). Parent involvement in education: toward an understanding of parents' decision making. The Journal of Educational Research, 100(13), 311-323. doi:10.3200/joer.100.5.311-323

Andrés, S. y Giró, J. (2016). El papel y la representación del profesorado en la participación de las familias en la escuela. Revista Electrónica Interuniversitaria de Formación del Profesorado, 19(1), 61-71. doi: http://dx.doi.org/10.6018/reifop.19.1.245461

Auduc, J. L. (2007). Les relations parents-enseignants à l'école primaire. Paris: CRDP de l'académie de Créteil.

Castro, M., Expósito, E., Lizasoain, L., López, E., y Navarro, E. (2014). Participación familiar y clima escolar en los centros de Educación Infantil, Educación Primaria y Educación Secundaria Obligatoria. En Consejo Escolar del Estado (Coord.), La participación de las familias en la educación escolar (pp. 181-193). Madrid: Ministerio de Educación, Cultura y Deporte.

Collet-Sabé, J., Besalú, X., Feu, J., y Tort, A. (2014). Escuelas, familias y resultados académicos. Un nuevo modelo de análisis de las relaciones entre docentes y progenitores para el éxito de todo el alumnado. Profesorado. Revista de currículum y formación del profesorado, 18(2), 7-33.

Epstein, J.L. (2001). School, Family, and Community Partnerships: Preparing Educators and Improving Schools. Boulder: Westview Press.

Feito, R. (2007). Balance de la participación de los padres en los consejos escolares de centro. Participación Educativa, 4, 4-15.

Garreta, J. (2013). La participación de las familias en la escuela: una cuestión pendiente, Documentación Social, 171,101-124.

Garreta, J. (Dir.) (2008). La participación de las familias en la escuela pública. Madrid: CIDE y CEAPA.

Hernández, M. A., García, M. P, Parra, J., y Gomariz, M. A. (2019). Perfiles de participación familiar en Educación Secundaria Obligatoria. Anales de Psicología, 35(1), 84-94. http://dx.doi.org/10.6018/analesps.35.1.325981- 84 - 
Hill, E. y Tyson, D. (2009). Parental involvement in middle school: a meta-analytic assessment of the strategies that promote achievement. Developmental Psychology, 45(3), 740-763.

Hoover-Dempsey, K. y Sandler, H. (1995). Parent involvement in children's education: why does it make a difference. Teachers College Record, 97, 310-331.

Inam, A., Nomaan, S., y Abiodullah, M. (2016). Parents' Parenting Styles and Academic Achievement of Underachievers and High Achievers at Middle School Level. Bulletin of Education and Research, 38(1), 57-74.

Jeynes, W. H. (2011). Parental Involvement and Academic Success. New York: Routledge.

Llevot, N. y Bernad, O. (2015). La participación de las familias en la escuela. Revista de la Asociación de Sociología de la Educación (RASE), 8(1), 57-70.

Lorenzo, M., Godás, A., y Santos Rego, M. A. (2017). Principales determinantes de la implicación y participación de las familias inmigrantes en la escuela. Cultura y Educación, 29(2), 213-253.

Lorenzo, M., Godás, A., Priegue, D., y Santos Rego, M. A. (2009). Familias inmigrantes en Galicia. La dimensión socioeducativa de la integración. Madrid: Ministerio de Educación.

Musitu, G. y Cava, M. (2001). La familia y la educación. Barcelona: Octaedro.

Parra, J., García, M.P., Gomariz, M.A., y Hernández, M.A. (2014). Perfiles de participación de las familias españolas en los centros educativos. En Consejo Escolar del Estado (Ed.). La participación de las familias en la educación escolar (pp. 127-148). Madrid: Ministerio de Educación, Cultura y Deporte.

Pomerantz, E., Moorman, E., y Litwack, S. (2007). The how, whom, and why of parents' involvement in children's academic lives: more is not always better. Review of Educational Research, 77(3), 373-410. doi: https://doi.org/10.3102/003465430305567

Reparaz, R. y Naval, C. (2014). Bases conceptuales de la participación de las familias. En Consejo Escolar del Estado (Coord.). La participación de las familias en la educación escolar (pp. 21-34). Madrid: Ministerio de Educación, Cultura y Deporte.

Rivera, M. y Milicic, N. (2006). Alianza familia-escuela: percepciones, creencias, expectativas y aspiraciones de padres y profesores de enseñanza general básica. PSYKHE-Escuela de Psicología, 15(1), 119-135. doi:10.4067/s0718-22282006000100010

Rodríguez, B., Martínez, R. A., y Rodrigo, M. J. (2016). Dificultades de las Familias para Participar en los Centros Escolares. Revista latinoamericana de educación inclusiva, 10(1), 79-98.

Roosa, M. W., O’Donnell, M., Cham, H., Gonzales, N. A., Zeiders, K. H., Tein, J., Knight, G. P., y Umaña-Taylor, A. (2012). A prospective study of mexican american adolescents' academic success: Considering family and individual factors. Journal of Youth Adolescence, 41, 307-319. 
Santos Rego, M. A., Godás, A., y Lorenzo, M. (2012). El perfil del alumnado repetidor y no repetidor en una muestra de estudiantes españoles y latinoamericanos: un estudio sobre los determinantes de sus logros académicos. Estudios sobre Educación, 23, 4362.

Santos Rego, M. A., Lorenzo, M., y Priegue, D. (2011). Infancia de la inmigración y educación: la visión de las familias. Revista de Investigación Educativa (RIE), 29(1), 97-110.

Santos Rego, M. A., Godás, A., y Lorenzo, M. (2016). ¿Puede la implicación de los padres mejorar el estudio de sus hijos en la escuela? La evidencia de un programa pedagógico. Estudios sobre Educación (ESE), 30, 9-30. doi: 10.15581/004.30.9-3

Silveira, H. (2016). La participación de las familias en los centros educativos. Un derecho en construcción. Revista Electrónica Interuniversitaria de Formación del Profesorado, 19(1), 17-29. doi: http://dx.doi.org/10.6018/reifop.19.1.245511 